\title{
Optical Characterization of Bi-pyramid Gold and Silver Nano- shells by Finite Difference Time Domain Method
}

\author{
Cherinet Seboka Ambaye ${ }^{1,2}$, Yunhu $\mathrm{Wu}^{1,3}$ Guoping Zhang ${ }^{1}$ \\ ${ }^{1}$ College of Physical Science and Technology, Central China Normal University, Wuhan 430079, China \\ ${ }_{2}^{2}$ (Department Physics, Madawalabu University, Bale Robe 217, Ethiopia) \\ ${ }^{3(}$ Department of Physics, Kashi Normal College, Kashi 844000,China)
}

\begin{abstract}
Optical characterization of both gold and silver bi-pyramid nano-shells are investigated using the finite difference time domain (FDTD) method. This method is a convenient way for describing both quantitatively and qualitatively the optical response of nano-oshells/nanoparticles of arbitrary geometry and shape that surrounding the active medium. The surface plasmon peaks of gold and silver bi-pyramid nano-shell can be tuned from visible to the near infrared region by controlling the size (aspect ratio), surface sharpness and polarization of light. Surface plasmons provide a powerful means of confining light to nano-shells, which in turn can enhance local electromagnetic fields and significantly amplify the signal finally used for surface enhanced Raman scattering (SERS).
\end{abstract}

Keywords: Finite Difference Time domain (FDTD), Surface enhanced Raman Scattering (SERS), Surface Plasmon Resonance(SPR), Enhancement of Local Electric Field.

\section{Introduction}

The optical properties of metalloid nanostructures are important from both a scientific and a technological perspective. In recent years, physical and chemical properties of metalloid nano-structure after interacting with optical light have received wider attentions in research and technological applications. The candidate metalloid nano-shells chosen for the investigation under in this paper are gold and silver bi-pyramids of various sizes. They possess a plasmon-derived tunable optical resonance controlled by the dimensions of the core (mainly silica core) and shell layers, spanning much of the visible and infrared region of the optical spectrum [1]. Additionally, nano-shell and other nano-scale metallic structures have been shown to greatly enhance local electromagnetic fields in certain regions near their surfaces at specific wavelengths of light, controlled by nano-structure geometry [1].

The linear and nonlinear optical response of metal nanoparticles is specified by oscillations of the surface electrons in the Coulomb potential formed by the positively charged ionic core. This type of excitation is called the surface plasmon (SP)[2]. In 1908 Mie proposed a solution of Maxwell's equations for spherical particles interacting with plane electromagnetic waves, which explains the origin of surface plasmon resonance (SPR) in the extinction spectra and coloration of metal colloids.

During the last century optical properties of NPs have extensively been studied and metal-dielectric nano-composites have found various applications in different fields of science and technology. When light is introduced into a nanometer scale object, such as a sub-wavelength aperture or a scatterer, a localized electromagnetic field (the optical near-field) is generated near the object. Since the optical properties of metal nanoparticles are governed by SPR, they are strongly dependent on the nano-particles' size, shape, concentration and spatial distribution as well as on the properties of the surrounding matrix. The energy intensity and the distribution of the optical near-field also depend on the object's shape, size, and dispersive material[3]. A broad range of applications using these nanoparticles in optical ranges have been studied for application of Raman spectroscopy, biological sensing, drug delivery materials, photo-thermal cancer therapy, [1].

A numerical approach is ultimately required to examine the optical properties of metalloid-dielectric nano-particles of arbitrary shape and symmetry. In this paper, we present our investigation of bi-pyramid nanoshells by using commercial software called CST microwave studio ${ }^{\circledR}$ which used for numerical simulation based on a finite difference time domain (FDTD) method. For characterization of optical response of gold and silver nano-shell with bi-pyramids structure is seen in figure 1 with some selected sizes as specified in table 1 . A plane wave of TEM mode is directed along axial direction or major axis where the incident waves scan the sharpest edges of the NPs. 


\section{Optical Pulse}
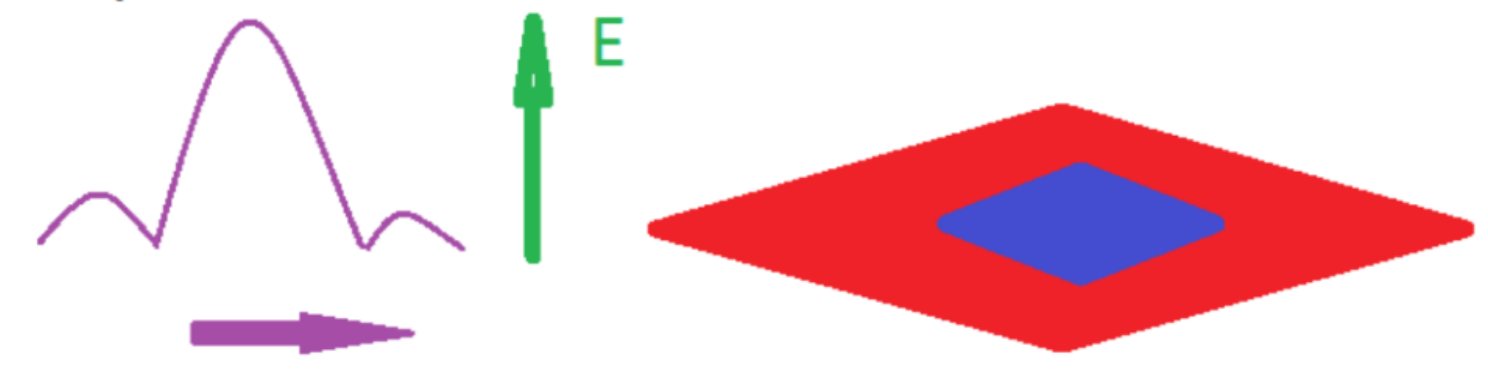

Figure 1: Au/Ag nano-shell bi-pyramid with silica core is immersed in a harmonic vertically oscillated electric field to generate Gaussian pulse directed along the major axis to induce surface plasmon resonance (SPR). The dielectric constant $\varepsilon_{h}$ of silica core represented by blue color is 2.08 .

II. Dielectric and Far-field Optical Response Functions of Nanoparticles

The electromagnetic response of metal is largely detected by the collective movement of free electron within the metal crystalline structure. The model for electron motion in metal is known as the Drude free electron model. With time varying harmonic incident electromagnetic wave, the equation of motion for free electron is given by

$$
m_{e f f} \frac{\partial^{2} \vec{r}}{\partial t^{2}}+m_{e f f} \Gamma \frac{\partial \vec{r}}{\partial t}=-e \vec{E}
$$

The frequency dependence of the dielectric function by intraband transition from eq(1) as

$$
\varepsilon_{\text {int } r a}=1-\frac{\omega_{p}^{2}}{\omega^{2}+i \Gamma \omega}
$$

Where $m_{\text {eff }}$ is the effective mass, $\Gamma$ is the electric damping or collision frequency in Drude electron model, $\omega_{p}=\sqrt{N e^{2} / \varepsilon_{0} m_{e f f}}$ is plasma frequency for the metal having $\mathrm{N}$ density of free electrons.

Although the behavior of noble metal is dominated by free electron response, the contribution from bound electron should also be taken to account when working with high frequencies in the visible ranges. As studies have shown that with high frequency transition to the bound electrons, it would carry out interband transition as similar to dielectric material expressed in classical Helmoltz-Drude model [14]

$$
\varepsilon_{\text {inter }}(\omega)=1+\sum_{j} \frac{S_{j} \omega_{j}^{2}}{\omega_{j}^{2}-\omega^{2}-i \Gamma_{j} \omega}
$$

Where $\omega_{j}$ is the resonance frequency of the $\mathrm{j}^{\mathrm{th}}$ mode. $\mathrm{S}_{\mathrm{j}}$ and $\Gamma_{j}$ represent the strength and the damping constant of the $\mathrm{j}^{\text {th }}$ mode respectively. Therefore, the overall the effective dielectric function of the metal is contributed from both interband transition due to bound electrons and intraband transition due to free electron given by

$$
\varepsilon(\omega)=\varepsilon_{\text {inter }}(\omega)+1-\frac{\omega_{p}^{2}}{\omega^{2}+i \Gamma \omega}
$$

According to Wenshan Cai and Vladimir Shalaev[14], the interband transition has a non-zero impact on dielectric function of the metal even at wavelength much longer than the resonance wavelength. When working at longer wavelengths away from the interband resonance (infrared range for gold, visible plus infrared for silver), we can replace the frequency-dependent $\varepsilon(\omega)$ in eq(4) by a constant offset denoted by $\varepsilon_{\infty}$. It is important to note that, due to the complicated band structure of bound electron in noble metals, there are usually multiple interband transitions in the UV or deep UV spectrum. Hence the offset $\varepsilon_{\infty}$ is the sum or integral value after taking all pertinent transition into account. Eventually, then, the modified Drude model taking into account the constant offset of interband transition becomes: 


$$
\varepsilon(\omega)=\varepsilon_{\infty}-\frac{\omega_{p}^{2}}{\omega^{2}+i \Gamma \omega}
$$

This Drude dielectric function [1] is fitted to a particular frequency range of the Jonson and Christy bulk dielectric data for the metal under study Corrections to the Drude dielectric function arising from the finite electron. For This Drude dielectric function was fitted to a particular frequency range of the Johnson and Christy bulk dielectric data for the metal under study. The numerical value used during CST microwave simulation used for gold is $\varepsilon_{\infty}=9.5, \omega_{p}=8.94 \mathrm{ev}, \Gamma=0.69 \mathrm{ev}$ and that of silver is $\varepsilon_{\infty}=5, \omega_{p}=9.5 \mathrm{ev}$, $\Gamma=0.0887$.

\section{Surface Plasmon Resonance}

The enchantment of gold nanoparticles since ancient times, as reflected in their intense color, originates from the basic photo-physical response that does not exist to nonmetallic particles. When a metal particle is exposed to light, the oscillating electromagnetic field of the light induces a collective coherent oscillation of the free electrons (conduction band electrons) of the metal. This electron oscillation around the particle surface causes a charge separation with respect to the ionic lattice, forming a dipole oscillation along the direction of the electric field of the light[4]. The amplitude of the oscillation reaches maximum at a specific frequency, called surface plasmon resonance (SPR)[4]. The SPR band is much stronger for plasmonc nanoparticles (noble metal, especially $\mathrm{Au}$ and $\mathrm{Ag}$ ) than other metals.

From the size dependence of the SPR, it is quite obvious that metal nanoparticles with nonspherical shape will show several LSPR in their spectra. For instance, the optical absorption spectrum of a collection of randomly oriented gold nanorods with aspect ratio R can be modelled using an extension of the Mie theory. Within the dipole approximation according to the Gans theory treatment [6], the extinction crosssection $\sigma_{\text {ext }}$ for ellipsoid like nanoparticle is given by the following equation when the light polarized along major axis $\mathrm{A}$

$$
\sigma_{e x t}=\frac{2 \pi V \varepsilon_{h}^{\frac{3}{2}}}{3 \lambda} \sum_{j} \frac{\left(1 / P_{A}^{2}\right) \varepsilon^{\prime \prime}}{\left(\varepsilon^{\prime}(\omega)+\frac{1-P_{A}}{P_{A}} \varepsilon_{h}\right)^{2}+\varepsilon^{\prime \prime}(\omega)^{2}}
$$

Where $\mathrm{V}$ is the volume of the particle, $P_{A}$ is defined as

$$
P_{A}=\frac{1-e^{2}}{e^{2}}\left[\frac{1}{2 e} \ln \left(\frac{1+e}{1-e}\right)\right]
$$

For the optical light polarized along minor axes B and $\mathrm{C}, P_{A}$ can either be replaced by $P_{B}$ or $P_{C}$ and such that

$$
P_{B}=P_{C}=\frac{1-P_{A}}{2}
$$

Where $e=\sqrt{1-\left(\frac{B}{A}\right)^{2}}=\sqrt{1-\frac{1}{R^{2}}}, R=\frac{A}{B}$ defines the aspect ratio.

Surface plasmon resonance (SPR) occurs for light polarized along major axis when the real part of the dielectric function $\varepsilon^{\prime}(\omega)$ satisfies the following condition as far as optical interaction of the imaginary dielectric function $\varepsilon^{\prime \prime}(\omega)$ in the denominator is weak or negligible.

$$
\varepsilon^{\prime}(\omega)=-\left(\frac{1-P_{A}}{P_{A}} \varepsilon_{h}\right)
$$

So in this paper we would like to examine both far and local field of gold and silver bi-pyramid nano-shell with silica cores of different sizes approximated as ellipsoid nano-shells for exploiting the above equations.

Assuming a homogeneous size distribution and therefore homogeneous line broadening mechanism seen in the figure 2 are related to Doppler, natural, and collision broadening. The finite life time of 
an excited state causes an uncertainty in the energy of the transition leading to natural broadening. As a consequence of the Heisenberg's uncertainty principle,

$$
\Delta E \Delta t \approx \hbar
$$

The energy level of an excited state of an atom or ion can never be determined precisely because its life time $\Delta t$ is finite. This, the line profile broadened over a natural line width at full width half maximum (FWHM) $\Delta \lambda_{1 / 2}[5]$ is given as

$$
\Delta \lambda_{1 / 2}=\frac{\lambda_{0}^{2}}{2 \pi c}\left[\frac{1}{\frac{1}{\Delta t_{i}}+\frac{1}{\Delta t_{f}}}\right]
$$

Where $\Delta t_{i}$ and $\Delta t_{f}$ are durations of the excited state at $\mathrm{i}^{\text {th }}$ and $\mathrm{f}^{\text {th }}$ of the particles. $\lambda_{0}$ is the wavelength at which surface plasmon resonance and the highest extinction spectra peak occurring. Thus effect leads to a Lorentz function as

$$
\phi(v)=\frac{\gamma}{4 \pi^{2}} \frac{1}{\left(v-v_{0}\right)^{2}+(\gamma / 4 \pi)^{2}}
$$

Where $\gamma$ is related to spontaneous decay rates $A_{i j}$ of excited states $\mathrm{j}$ into lower states $\mathrm{i}$ through

$$
\gamma=\sum_{j} A_{j i}
$$

The decay rates $A_{i j}$ result from quantum mechanical treatment of the respective transitions and can be found in standard tables.

\section{Discussion Results}

When a metal particle is exposed to light, the oscillating electromagnetic field of light induces a collective coherent oscillation of the electrons (conduction band electrons) of the metal. These electron oscillations around the particle surfaces cause charge separations with respect to the ionic lattice, forming a dipole oscillation of the electric field of the light. The amplitude of the oscillation reaches maximum at specific frequency, called surface plasmon resonance (SPR)[4]. The surface plasmon resonance of the NPs depends on the shape, size, deformity and direction of polarization of the incident wave.

The settled and some statistical processed data that we see in table 1 are obtained from the technique of curve fitting after simulation results. One of the variable that comparing the degree of coherency during state transition from i state to $f$ state measured in the order of femto $\left(10^{-15}\right)$ seconds are calculated according eq(11)

$$
\frac{1}{\Delta t}=\frac{1}{\Delta t_{i}}+\frac{1}{\Delta t_{f}}=\frac{\Delta \lambda_{1 / 2} 2 \pi c}{\lambda_{0}^{2}}
$$

Table 1: The settled and the processed data of Ag/ Au Bi-pyramid nano-shells with silica cores

\begin{tabular}{|l|c|c|c|c|c|c|c|c|c|}
\hline $\mathrm{NP}$ & $\mathrm{SR}$ & $\mathrm{CR}(\mathrm{nm})$ & LHS $(\mathrm{nm})$ & LHC $(\mathrm{nm})$ & $\lambda_{0}(\mathrm{~nm})$ & $\mathrm{SD}(\mathrm{nm})(\sigma)$ & FWHM(nm) & $\Delta t(\mathrm{fs})$ & $\mathrm{R}$ \\
\hline$\left(\mathrm{AgSi} 0_{2}\right)_{1}$ & 30 & 20 & 100 & 80 & 516.88 & 18.05 & 42.50 & 3.14 & 1.67 \\
\hline$(\mathrm{AgSiO})_{2}$ & 30 & 20 & 60 & 40 & 461.20 & 10.60 & 24 & 4.76 & 1 \\
\hline$\left.(\mathrm{AgSi})_{2}\right)_{3}$ & 35 & 25 & 70 & 50 & 487.47 & 15.29 & 35.99 & 3.2 & 1 \\
\hline$\left(\mathrm{AgSi} 0_{2}\right)_{4}$ & 40 & 30 & 80 & 60 & 512.47 & 14.11 & 33.24 & 4.2 & 1 \\
\hline$\left(\mathrm{AuSi}_{2}\right)_{1}$ & 30 & 20 & 60 & 40 & 540 & 9.33 & 21.98 & 7.04 & 1 \\
\hline$\left(\mathrm{AuSiO}_{2}\right)_{2}$ & 35 & 25 & 70 & 50 & 560 & 10.54 & 24.83 & 6.7 & 1 \\
\hline$\left(\mathrm{AuSi} 0_{2}\right)_{3}$ & 40 & 30 & 80 & 60 & 582 & 11.90 & 28.02 & 6.45 & 1 \\
\hline$\left(\mathrm{AuSi}_{2}\right)_{4}$ & 30 & 20 & 100 & 80 & 587.83 & 15.63 & 36.80 & 5.0 & 1.67 \\
\hline
\end{tabular}

In table 1 , there are lists of the size of the shell radius (SR), core radius (CR), the lateral height of the shell (LHS), and the lateral height of the core (LHC) of the bi-pyramid nano-shells seen in figure 1. The standard deviation $\mathrm{SD}(\sigma)$ and full width at half maximum FWHM are statistical tools for measuring the spread of the energy levels during transitions from higher states to the lower states. These statistical tools are extracted from the absorption or extinction spectra of NPs at the central wavelength $\lambda_{0}$ within optical frequency range. 
Particle with larger aspect ratio $\mathrm{R}$ would experience surface plasmon absorption to red shift or lower energy level. On the other hand a particle with relatively small aspect ratio $\mathrm{R}$ would experience surface plasmon to the blue shift or higher energy level seen in figure 2.

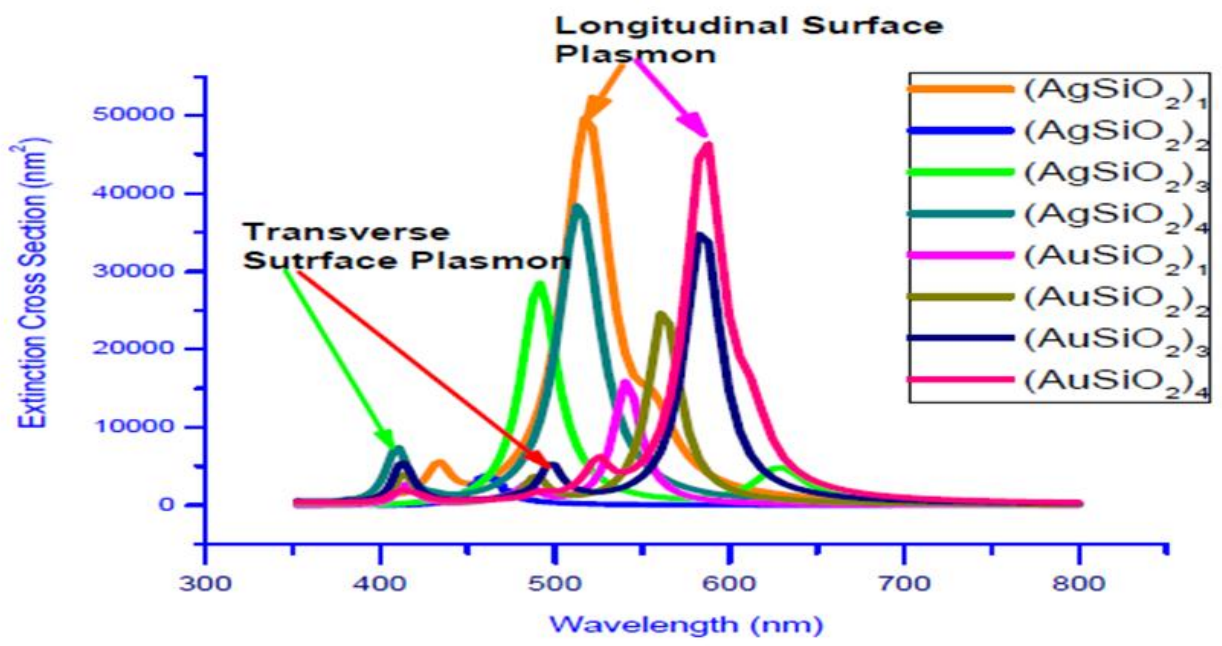

Figure 2: Extinction (absorption plus scattering) spectra of bi-pyramid gold and silver nano-shell with silica core.

Stephen Link and Mostafa. El-Sayed[6] on their review literature discussed the split of resonance absorption band in two bands when gold NPs become more elongated along one axis. The aspect ratio is the value of the long axis (length) divided by short axis (width) of a cylindrical or rod shaped particle. As the aspect ratio increases, the energy separation between the resonance frequencies of the two plasmon bands increases. The short-wavelength extinction band due to the oscillation of the electrons perpendicular to the axial distance (major axis) of bi-pyramid nano-shells is termed as transverse plasmon while the longer-wavelength band caused by the oscillation along the axial distance (major axis) is termed as longitudinal plasmon.

In addition to the scattering and absorption of light, sharp and pointed edges of gold or silver nanostructures can be used to substantially enhance local electromagnetic fields[7]. Upon excitation of surface plasmons (SPs), charges will be concentrated at the metal-dielectric interface, resulting in very strong amplification of the electric field [8]. The ability of sharped edged of gold and silver nanostructures enhancing the local electric fields dramatically has led to the development of efficient surface-enhanced spectroscopy[7$10]$.

Referring to figure 3, among the lists of NPs from silver family in table 2, the enhancement of local electric field of $(\mathrm{AgSiO} 2)_{1}$ is boosted by more than 4000 factors. This is because of the two opposite edges of $(\mathrm{AgSiO})_{1}$ is sharper than the rest of the member of the silver family.
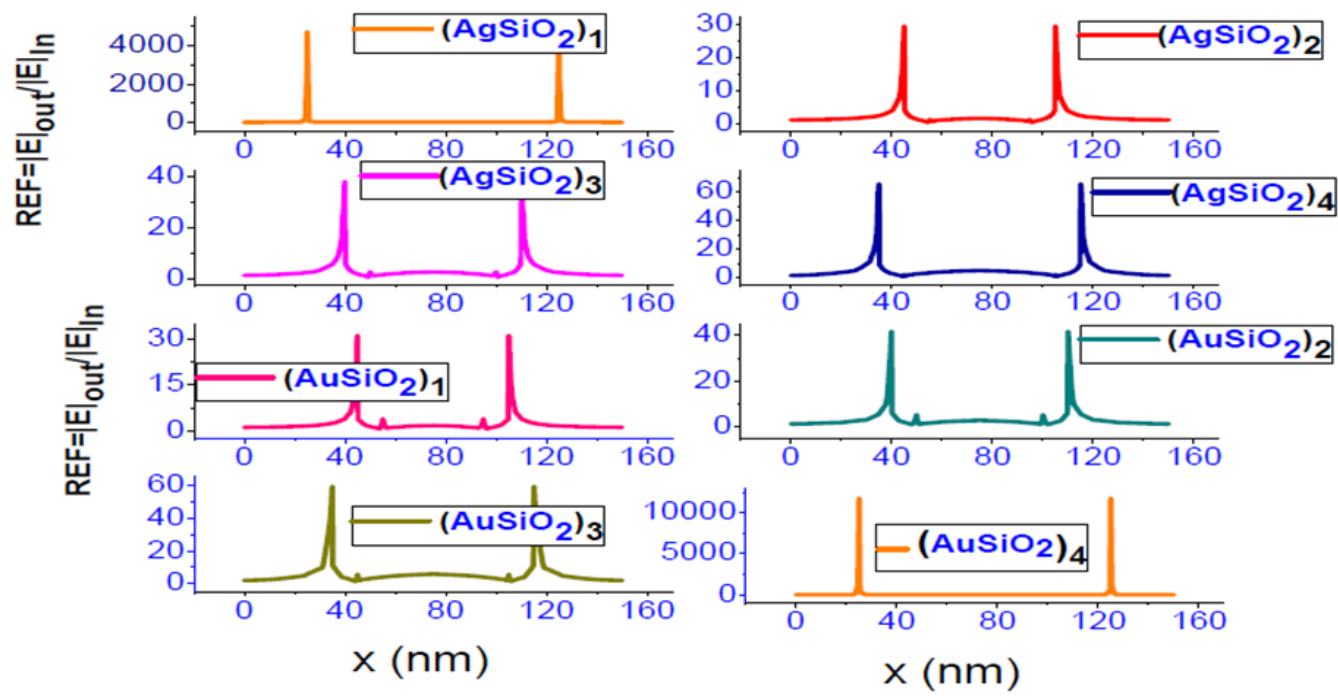
Figure 3: The relative electric field from the surface of NPs of silver and gold families versus the curve length along $\mathrm{x}$ direction. The specification of the NPs are found in table 2.

On the similar characterization, among the list of NPs from gold family in table 1, the enhancement of local electric field of $(\mathrm{AuSiO} 2)_{4}$ is aggressively boosted by the factor of more than 12,500 compared to other member of gold family. This property of NPs either from gold or silver family have attracted attenuation for development of surface-enhanced Raman spectroscopy or surface-enhanced Raman scattering (SERS) for detection of molecules with high resolution $[11,12,13]$. The weak Raman signal can be greatly enhanced by the introduction of SERS spectroscopy in 1974 by Fleischmann et al.[11] from the University of Southampton. It was accidentally discovered by them when they tried to do Raman with an adsorbate of very high Raman crosssection, such as pyridine (Py) on the roughened silver (Ag) electrode. The initial idea was to generate high surface area on the roughened metal surface.

\section{Conclusion}

We have studied the general behavior of optical responses, the surface plasmon resonances and enhancement of local electric field from gold and silver bi-pyramid nano-shell with silica core by finite difference time domain method using CST microwave studio ${ }^{\circledR}$ simulation. Theoretical exploration on optical response in nano-metallic structure would contribute for improvement of spectroscopic method for current and future studies. Sharper Edges and distorted surfaces enhance electric field at large in comparison with the lateral or smooth structures. The optical tunablities from different gold/silver nanostructures are well suited for various applications like biomedical sensing, fluorescent bio-imaging, chemical sensing for monitoring the environment and so on. These are also important for applications like cancer diagnosis and photo-thermal therapy involving metallic NPs as sensors. Noble metal such as silver and gold NPs exhibit sharp spectral extinction peaks at visible and nearinfrared frequencies due to the resonant excitation of their free electrons termed as localized surface plasmon resonance (LSPR). It has been found out that sharp and distorted surfaces have more sensitive to the polarization of light to produce plasmon resonances in a wider energy range. Moreover from the simulation results lower size bi-pyramid NPs have higher degree of transition coherency and stability than the larger NPs during the occurrence of surface plasmon resonances.

\section{Acknowledgements}

I would like to appreciate Professor Guoping Zhang and Dr. Yunhu Wu for professional assistance and consultation from Central China Normal University (CCNU) at College of Physical Science and Technology in the Lab of Optoelectronics and Information Engineering during my stay in China.

\section{References}

[1]. Chris Oubre, Peter Nordlander Optical, "Properties of Metallodielectric Nanostructures Calculated Using the Finite Difference Time Domain Method", J. Phys. Chem. B (2004), 108, 17740-17747

[2]. A. Stalmashonak, "Optical Properties of Nanocomposites Containing Metal Nanoparticles," Metal-Glass Nanocomposites, SpringerBriefs in Physics, 2013

[3]. Takashi Yamaguchi and Takashi Hinata, "Optical near-field analysis of spherical metals: Application of the FDTD method combined with the ADE method," OPTICS EXPRESS 11481, Vol. 15, No. 18, 3 September 2007

[4]. Xiahua Huang, Mostafa A. El-Sayed, "Gold nanoparticle: Optical Properties and Implementations in Cancer Diagnosis and Photthermal Therapy", Journal of Advanced Research (2010) 1, 13-18

[5]. J.COOPER, "Plasma spectroscopy-Plasma Physics Group, Imperial College, London" Iopscience.jop.org,(http://iopscience.iop.org/0034-4885/29/1/302)

[6]. S. Link, M. B. Mohamed, M. A. El-Sayed, "Simulation of the Optical Absorption Spectra of Gold Nanorods as a Function of Their Aspect Ratio and the Effect of the Medium Dielectric Constant", J. Phys. Chem. B 1999, 103, 3073-3077 3073

[7]. Cecilia Noguez, "Surface Plasmons on metal nanoprticle: The influence of shape and physical Envirnment", J. Phys. Chem. C 2007, 111.3806-3819

[8]. Younan Xia and Naomi J. Halas, "Shape-Controlled Synthesis and Surface Plasmonic Properties of Metallic Nanostructures", Mrs Bulletin Volume 30 May 2005,338-348, [www.mrs.org/publications/bulletin]

[9]. Reshim Thomas, Jatish, kumar, R.S.Swathi, KGeorge Thomas, "Optical Effects near metal nanostructures: towards surfaceenhanced spectroscopy", Current Science, VOL. 102, NO. 1, 10 JANUARY 2012

[10]. A.Stalmashonak, "Optical properties of Nanocomposites Containg metal nanoparticles", SpringerBriefs in Physics (2013) 5-15

[11]. Ujjal Kumar Surl, and Joydeep Chowdhury, "Surface-enhanced Raman scattering: overview of a versatile technique used in electrochemistry and nanoscience", CURRENT SCIENCE, VOL. 105, NO. 7, (10 OCTOBER 2013), 923-939

[12]. Hyunhyub Ko, Srikanth Singamaneni, and Vladimir V. Tsukruk, "Nanostructured Surfaces and Assemblies as SERS Media", 2008 Wiley-VCH Verlag GmbH \& Co. KGaA, Weinheim, No. 10, 1576-1599, [www.small-journal.com]

[13]. Nastaran Kazemi-Zanjani, Sylvain Vedraine, Franois Lagugne-Labarthet, "Localized enhancement of electric field in tip enhanced Raman spectroscopy using radially and linearly polarized light”, Optical Society of America, (21 October 2013), Vol. 21, No. 21,[DOI:10.1364/OE.21.025271-OPTICS EXPRESS 25271]

[14]. W.Cai, V Shalaev, "Optical Metamterial: Fundamntal and Application, Springer Science+Business Media, Stanford \& Prude University, USA, (2010), (19-36) 